Canadian Oncology

Nursing Journal

Revue canadienne

de soins infirmiers

en oncologie

Volume 28, Issue 4 • Fall 2018

elSSN: 2368-8076 


\section{Développement d'un consensus professionnel face aux interventions de l'infirmière pivot en oncologie en vue de soutenir la planification des effectifs au Québec}

\section{par Andréanne Saucier et Alain Biron}

RÉSUMÉ

Au Québec, près de 250 infirmières pivot en oncologie (IPO) sont déployées au Québec pour soutenir les personnes atteintes d'un cancer. Il devient toutefois de plus en plus évident que ce nombre est insuffisant. Une étude a été menée afin d'estimer les besoins en IPO pour la province. L'établissement d'un consensus professionnel par la technique du groupe nominal et la méthode Delphi ont été utilisés pour atteindre cet objectif. Les données obtenues permettent de baser les décisions de planification des effectifs sur des évidences mesurées dans la réalité du travail actuel des IPO. Ainsi, il est espéré que celles-ci contribueront à une meilleure planification des effectifs pour répondre aux besoins de personnes atteintes de cancer et leurs proches.

\section{CONTEXTE}

L es infirmières pivot en oncologie (IPO) ont été introduites ـen 2005 au Québec (Comité de l'évolution de la pratique infirmière en oncologie [CEPIO], 2005; Fillion et al., 2009; Fillion et al., 2010; Plante et Joannette, 2009). Ces infirmières sont des personnes-ressources pour les patients atteints de cancer et leurs proches en intervenant dès l'annonce du diagnostic et tout au long de la trajectoire de la maladie. Un des objectifs premiers de l'introduction de ce rôle était de diminuer la fragmentation des soins. Plus spécifiquement, leur rôle unique consiste à évaluer, enseigner et informer, soutenir, coordonner les soins pour s'assurer de leur continuité (DLCC, 2008).

\section{AU SUJET DES AUTEURS}
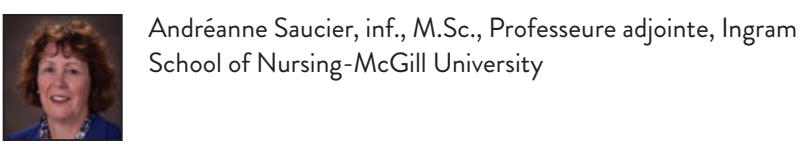

Andréanne Saucier, inf., M.Sc., Professeure adjointe, Ingram School of Nursing-McGill University

Alain Biron, inf., Ph.D., Directeur adjoint des soins infirmiers, Volet pratique professionnelle, Professeur adjoint, Ingram School of Nursing-McGill University

Auteur-ressource : Alain Biron, Centre universitaire de santé McGill, 1650, avenue Cedar, Montréal (Québec) H3G 1A4 Courriel : alain.biron@muhc.mcgill.ca

Les auteurs tiennent à remercier la Direction générale de cancérologie pour leur soutien financier ainsi que leur appui à la réalisation de l'étude. Les auteurs aimeraient aussi remercier Catherine Nesbitt, coordonnatrice de l'étude et Myriam Skrutkowski pour son soutien dans le développement des stratégies basées sur les résultats de l'étude.

DOI:10.5737/23688076284301307
Au Québec, plus de 49100 personnes reçoivent un diagnostic de cancer chaque année (Comité consultatif des statistiques canadiennes sur le cancer de la Société canadienne du cancer, 2014). Près de 250 IPO ont été déployées dans toutes les régions du Québec pour soutenir ces personnes (Direction de la lutte contre le cancer [DLCC], 2011). Il avait été initialement prévu lors de ce déploiement d'atteindre un ratio de 1 IPO par 200 nouveaux patients atteints de cancer. C'est ce ratio qui a été utilisé pour planifier le nombre d'IPO nécessaires au niveau provincial à ce moment (DLCC, 2007). Malgré l'augmentation des nouveaux cas de cancer, le nombre d'IPO n'a pas beaucoup varié depuis 2011. Il devient toutefois de plus en plus évident que ce nombre est probablement insuffisant. De fait, ce ratio n'a jamais été atteint au cours des dernières années.

Outre le nombre de personnes diagnostiquées par année, une estimation des effectifs requis nécessite une connaissance $\mathrm{du}$ niveau de soins requis par les patients et leurs familles ainsi que la capacité réelle (productivité) de suivi des infirmières pivot en oncologie (Birch et al., 2007). Une étude de temps et mouvement a permis d'obtenir l'information nécessaire sur la productivité (Biron et Saucier, 2012. Cette étude a précisé le temps consacré aux tâches connexes inhérentes à l'organisation du travail (tâches non productives et tâches administratives). Il s'avère que 22,4 \% du temps de l'IPO est consacré à ces tâches connexes qui sont inhérentes à l'organisation du travail (Biron et Saucier, 2012).

Cependant, le niveau de soins requis des patients n'est pas encore connu, limitant ainsi une planification plus rigoureuse de la main-d'œuvre requise. Différentes méthodes ont été utilisées pour déterminer le niveau de soins requis en sciences infirmières (Spetz, 2008). Ces méthodes ont été pour la plupart développées dans le contexte de patients hospitalisés. Il faut alors se tourner vers d'autres options en l'absence de méthodes éprouvées, considérant l'absence de méthode validée par la recherche pour les infirmières œuvrant en contexte ambulatoire. Le consensus professionnel en utilisant la technique du groupe nominal (TGN) ou Delphi constitue une avenue possible dans les situations où les données probantes sont absentes. Ces techniques permettent d'établir un consensus professionnel de manière efficace (Hasson et Keeney, 2011). Il serait alors possible d'établir un consensus professionnel afin de déterminer les besoins des patients à travers l'identification des interventions infirmières jugées essentielles ainsi que le temps requis pour les effectuer. 
Dans ce contexte, l'objectif de cette étude consiste à établir un consensus professionnel relatif aux interventions infirmières jugées essentielles ainsi que le temps requis pour les effectuer. Les données recueillies permettront de combler les informations requises pour une meilleure planification des effectifs IPO au niveau du Québec. Ultimement, une meilleure planification permettra d'assurer un accès plus équitable aux patients atteints de cancer tout en minimisant les impacts négatifs qu'une sous-estimation pourrait avoir sur les infirmières pivot et les patients.

\section{MÉTHODE}

La méthode se divise en deux phases. La première phase consiste en l'application de la technique du groupe nominal (TGN) (Harvey et Holmes, 2012). La deuxième phase, la méthode Delphi (Hasson et Keeney, 2011), utilise les résultats de la technique du groupe nominal pour informer les éléments faisant l'objet de la méthode Delphi afin d'obtenir un consensus auprès des toutes les IPO du Québec relatif aux interventions jugées essentielles et le temps requis pour les compléter. La méthode sous-jacente à ces deux phases est décrite ci-après.

\section{Technique du groupe nominal}

Dans ce projet, un groupe d'IPO expérimentées, reconnues comme expertes par leurs pairs, ont été appelées à générer une liste d'interventions jugées essentielles et à établir un consensus initial sur le temps requis pour effectuer ces interventions.

Sélection du groupe d'experts. Un échantillon de convenance de 12 IPO expertes a été utilisé. Léquipe de recherche a contacté les personnes responsables au niveau des Agences de la santé et des services sociaux et des Centres hospitaliers universitaires (CHU) afin de recruter cet échantillon. Chaque personne responsable a nommé au moins une infirmière ayant au moins 3 ans d'expérience comme IPO et reconnue comme experte. Le tirage au sort a été utilisé lorsque plus de 4 experts d'une même désignation (locale, régionale et suprarégionale) désiraient prendre part au projet afin d'assurer une représentation équitable des différentes désignations.

Procédure. Après la sélection des experts, une journée de travail a été organisée. Ces IPO ont reçu au préalable une lettre leur décrivant la TGN et les invitant à réfléchir aux interventions/activités jugées professionnellement importantes dans leur rôle d'IPO ainsi que le temps requis pour les effectuer en considérant les différentes étapes de la trajectoire de la maladie à savoir : l'annonce du diagnostic de cancer et la préparation au premier traitement, les traitements de chirurgie, les traitements de chimiothérapie, les traitements de radiothérapie, le suivi post-traitements et la transition des soins curatifs vers les soins palliatifs.

La journée a débuté par une introduction qui venait préciser certaines informations transmises au préalable. Par la suite, et pour les cinq étapes de la trajectoire, les infirmières expertes ont généré en silence une liste d'interventions potentielles. Les IPO avaient comme instruction de se baser sur leur expérience et leur avis professionnel de ce que devraient être les interventions de l'IPO pour bien répondre aux besoins des patients atteints de cancer et leurs proches.
Un maximum de 5 interventions par étape de la trajectoire de la maladie pouvaient être retenues. Il fallait aussi préciser le nombre de minutes jugé adéquat pour chacune des interventions sélectionnées, toujours en se référant à une situation type. Le temps devait inclure la documentation. Ces interventions ont été par la suite partagées en groupe une fois identifiées lors de cette première étape (génération des idées en silence). Cette étape a été suivie d'une période de discussion et de clarification. La dernière étape était un vote secret électronique pour prioriser les interventions trouvées les plus importantes. Cette procédure suit l'approche classique de la technique du groupe nominal (Harvey et Holmes, 2012).

\section{Méthode Delphi}

Sélection des participants. Léquipe de recherche a obtenu la permission de la directrice adjointe de la DLCC de contacter directement les infirmières pivot pratiquant au Québec et ayant une adresse courriel valide soumise à cette direction. De par ce fait, la population entière d'IPO au Québec a été sélectionnée.

Questionnaires. La solution en ligne « SurveyMonkey ${ }^{\mathrm{TM}}$ » a été utilisée pour élaborer le questionnaire à partir des 29 interventions sélectionnées lors de la TGN. Les participantes devaient répondre aux questions par « Oui, je suis d'accord / Non, je ne suis pas d'accord » en se basant sur leur expertise quant au temps requis pour bien faire compléter l'intervention et bien répondre aux besoins d'un patient type, soit ni trop complexe, ni trop simple. Les participantes devaient proposer une réponse alternative parmi un choix préétabli dans l'éventualité où elles n'étaient pas en accord avec l'énoncé proposé. De plus, et ce pour chacune des phases de la trajectoire de la maladie, les participantes devaient aussi répondre à la question suivante : «Dans votre rôle d'IPO, intervenez-vous auprès des patients durant la phase de... ». À la fin du questionnaire, des informations de nature démographique étaient demandées.

Un pré-test auprès de quatre (4) IPO a été effectué afin de valider la compréhension du questionnaire en utilisant la technique d'entrevue cognitive (Presser et al., 2004). Ces entrevues ont permis d'apporter des changements au questionnaire pour assurer sa compréhension avant une plus large diffusion. Finalement, une question était jugée avoir obtenu un consensus lorsque $65 \%$ et plus des répondantes étaient en accord avec l'énoncé. Si le consensus était insuffisant après le premier questionnaire, une autre ronde de consultation était alors effectuée jusqu'à l'obtention du consensus. Une lettre d'introduction fournissait alors un résumé des énoncés pour lesquels un consensus avait été atteint. Seules les participantes à la première ronde étaient sollicitées pour participer à cette deuxième ronde.

Analyse. Des statistiques descriptives ont été utilisées pour déterminer la présence ou l'absence d'un consensus pour chaque énoncé du questionnaire Delphi. Les résultats de la méthode Delphi sont par la suite utilisés dans les équations sous-jacentes au calcul des effectifs nécessaires en tenant compte des paramètres suivants : le nombre de nouveaux cas par année, la cible d'accès au Québec au moment de l'étude 
(70\%), le temps dédié aux facteurs organisationnels $(22,4 \%)$, et en utilisant 1885 heures par année pour estimer les besoins en équivalent à temps complet (ETC).

Éthique. Le protocole élaboré pour la recherche de consensus avec la technique du groupe nominal et la méthode Delphi a été soumis au Comité d'évaluation de la recherche (CÉR) de la Faculté de médecine de l’Université McGill.

\section{RÉSULTATS}

Les résultats sont présentés pour chacune des deux phases de cette étude.

\section{Technique du groupe nominal}

Les participantes sélectionnées comme expertes avaient en moyenne près de 30 ans d'expérience, dont 5 ans comme IPO; une très grande majorité d'entre elles possédaient un baccalauréat et travaillaient à temps plein. Ces données viennent appuyer la sélection du groupe d'experts. Qui plus est, l'objectif d'avoir une représentation équitable entre les différentes désignations a été atteint.

Le groupe d'expert a identifié 29 interventions jugées prioritaires selon les différentes étapes de la trajectoire de la maladie ainsi que le temps requis pour chacune de ces interventions. Pour chacune des étapes de la trajectoire de la maladie, cinq (5) interventions ont été retenues à l'exception de la phase "Suivi post-traitements », où seulement quatre interventions ont été retenues. Un consensus a été atteint quant au choix des interventions. Un consensus a aussi été atteint sur la nature des interventions requises pour chaque étape de la trajectoire de la maladie. Cependant, il n'y a pas eu de consensus parmi ce groupe d'expert sur le temps requis pour effectuer ces interventions. Cette liste d'interventions ainsi que le temps requis a été utilisée pour élaborer le questionnaire de la méthode Delphi.

\section{Méthode Delphi}

Un total de 252 questionnaires ont été acheminés aux IPO par voie électronique et 122 ont été retenus pour fin d'analyse, ce qui représente un taux de réponse de $48 \%$. Les caractéristiques sociodémographiques des répondantes pour le premier tour sont présentées au tableau 1 . Suite à la compilation du questionnaire, 16/29 interventions de l'IPO ont atteint le pourcentage d'accord préétabli pour déterminer la présence d'un consensus. La décision a été prise de préparer une deuxième ronde pour tenter détablir un consensus pour les 13 interventions pour lesquelles le consensus n'avait pas été obtenu à la première ronde.
À la deuxième ronde de la méthode Delphi, le taux de réponse a été de $68 \%$, soit 83/122 questionnaires reçus. Les résultats révèlent que le consensus a été atteint pour le temps requis afin d'accomplir 7 des 13 interventions soumises lors de la deuxième ronde. Comme le temps requis pour accomplir les six autres interventions n'a toujours pas atteint un consensus parmi les participantes ayant répondu et que les pourcentages obtenus pour ces interventions sont très semblables à la première ronde, il a été décidé d'arrêter le processus (Boulkedid, Abdoul, Loustau, Sibony et Alberti, 2011).

Le pourcentage d'accord lors de la TGN et pour les deux rondes de la méthode Delphi pour la première étape de la trajectoire de la maladie, annonce du diagnostic, est illustré à titre d'exemple au tableau 2 (les résultats spécifiques pour chaque étape de la trajectoire de la maladie sont disponibles sur demande). Le temps requis à l'IPO pour une étape de la trajectoire de la maladie correspond à la somme du temps requis pour chaque intervention jugée nécessaire pour cette étape. Le tableau 3 présente la somme du temps requis pour chaque étape de la trajectoire de la maladie. Le temps requis pour accomplir 6 des 29 interventions ne faisait pas l'objet d'un consensus après deux rondes. Dans cette situation, la valeur inférieure et la valeur supérieure entre lesquelles la plus grande proportion des répondantes se situe sont utilisées. La présence d'un intervalle de temps au tableau 3 indique le fait que le temps requis pour accomplir l'une de ces interventions n'a pas fait l'objet d'un consensus dans cette étape.

Il faut se rappeler que chaque patient a sa propre trajectoire : par exemple, un patient peut ne pas avoir besoin de traitement de radiothérapie ou n'aura pas besoin de soins palliatifs. Chacune des phases de la trajectoire de la maladie est un tout en soi et on ne peut additionner que les phases spécifiques à chaque patient.

Ces données nous informent sur la situation actuelle de ce groupe d'IPO et sont des pistes de réflexion pour la planification des effectifs. On constate que les IPO sont très impliquées dès l'annonce du diagnostic, lors des traitements de chimiothérapie, en suivi post-traitements ou lors de la transition vers les soins palliatifs; elles le sont beaucoup moins lors des soins chirurgicaux et des traitements de radiothérapie.

Le pourcentage $(22,4 \%)$ est utilisé pour quantifier le temps consacré aux tâches connexes inhérentes à l'organisation du travail (tâches non cliniques) et doit être ajouté aux résultats obtenus par la méthode Delphi. Finalement, à

Tableau 1 : Caractéristiques des répondantes - Méthode Delphi

\begin{tabular}{|l|c|c|c|c|c|c|c|}
\hline Désignation & & \multicolumn{3}{|c|}{ Expérience (en années) } & \multicolumn{3}{c|}{ Formation } \\
\hline & N & Infirmière & Oncologie & IPO & Baccalauréat & Maîtrise & CSIO(C) \\
\hline Locale & 49 & Min. 15 ans & $5-6$ ans & $4-5$ ans & $88 \%$ & $2 \%$ & $26,5 \%$ \\
\hline Régionale & 21 & 10 ans & $5-6$ ans & 4 ans & $95 \%$ & $5 \%$ & $38 \%$ \\
\hline Suprarégionale & 52 & $10-15$ ans & $8-9$ ans & $4-5$ ans & $88 \%$ & $12 \%$ & $52 \%$ \\
\hline Total & 122 & & & & $89 \%$ & $6,5 \%$ & $39 \%$ \\
\hline
\end{tabular}


Tableau 2 : Niveau d'accord sur l'intervention et le temps requis pour la phase annonce du diagnostic

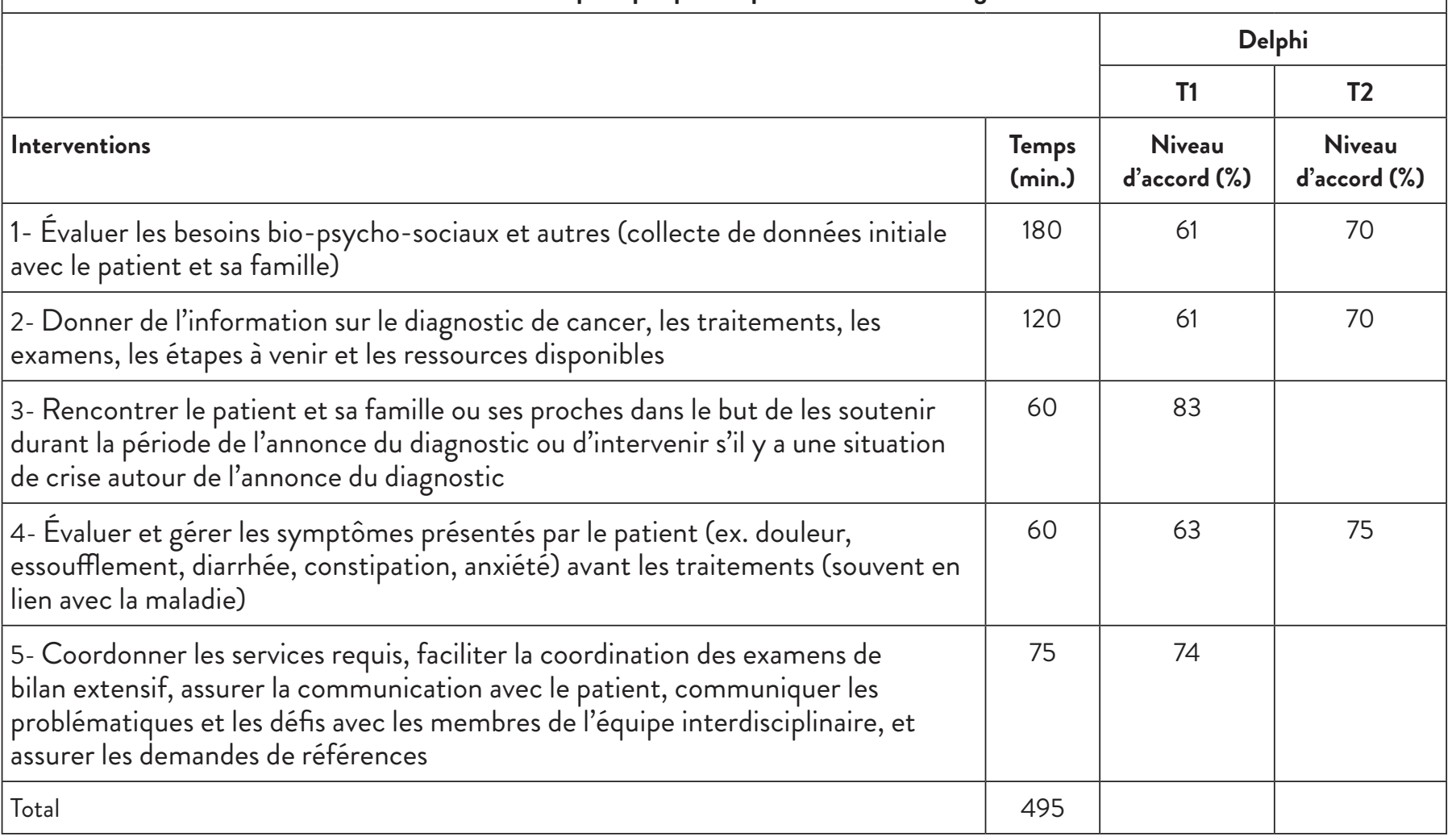

Tableau 3 : Résultats du consensus d'experts exprimés en minutes

\begin{tabular}{|l|c|}
\hline Trajectoire de soins & Temps (min) \\
\hline Annonce du diagnostic & 495 \\
\hline Soins chirurgicaux & 270 \\
\hline $\begin{array}{l}\text { Traitements de } \\
\text { chimiothérapie }\end{array}$ & $1080-1140$ \\
\hline $\begin{array}{l}\text { Traitements de } \\
\text { radiothérapie }\end{array}$ & $220-320$ \\
\hline Suivi post-traitements & $165-195$ \\
\hline $\begin{array}{l}\text { Transition vers les soins } \\
\text { palliatifs }\end{array}$ & $270-420$ \\
\hline Total & $\begin{array}{c}\text { Min. } 2500- \\
\text { Max. 2840 }\end{array}$ \\
\hline
\end{tabular}

ces données, il faut ajouter des informations de nature épidémiologiques quant au nombre de nouveaux patients par année en fonction des phases de la trajectoire de soins. Ces données proviennent de différentes sources. L'estimation doit aussi tenir compte de la cible ministérielle, à savoir que $70 \%$ des nouveaux cas devraient avoir accès à une IPO. De plus, nous avons utilisé 1885 heures comme base de calcul pour un poste d'IPO à temps complet (ETC). Selon ces paramètres, l'équation utilisée pour estimer les effectifs requis devient :
Tableau 4. Proportion des infirmières pivot impliquée par phase de soins les niveaux local, régional et suprarégional

\begin{tabular}{|l|c|c|c|c|}
\hline Phase & Local & Régional & Suprarégional & Total \\
\cline { 2 - 5 } & $\%$ impliquées & $\%$ impliquées & $\%$ impliquées & $\%$ impliquées \\
\hline Annonce du diagnostic & 100 & 100 & 100 & 100 \\
\hline Soins chirurgicaux & 65 & 52 & 62 & 61 \\
\hline $\begin{array}{l}\text { Traitements de } \\
\text { chimiothérapie }\end{array}$ & 93 & 100 & 96 & 96 \\
\hline $\begin{array}{l}\text { Traitements de } \\
\text { radiothérapie }\end{array}$ & 49 & 67 & 79 & 67 \\
\hline Suivi post-traitements & 97 & 95 & 88 & 93 \\
\hline $\begin{array}{l}\text { Transition vers les soins } \\
\text { palliatifs }\end{array}$ & 96 & 100 & 100 & 98 \\
\hline
\end{tabular}

Nombre ETC $=$ Nombre de nouveaux cas $\times 70 \% \times\left(\right.$ temps requis $\left.\left(\frac{\mathrm{min}}{\text { cas }}\right)+22,4 \%\right)$

$$
\div 60 \frac{\min }{\mathrm{Hr}} \div 1885 \frac{\mathrm{Hr}}{E T C}
$$

A titre d'exemple pour la phase « annonce du diagnostic » :

$$
\begin{aligned}
\text { Nombre ETC } & =46400 \text { nouveaux cas } \times 70 \% \times\left(495 \min \left(\frac{\min }{\text { cas }}\right)+22,4 \%\right) \\
& \div 60 \frac{\mathrm{min}}{\mathrm{Hr}} \div 1885 \frac{\mathrm{Hr}}{\mathrm{ETC}}
\end{aligned}
$$

Nombre ETC $=173,4$ 


\section{DISCUSSION}

Lobjectif de cette étude était d'établir un consensus professionnel précisant les interventions de l'infirmière pivot en oncologie jugées essentielles pour les patients tout au long de la trajectoire de la maladie ainsi que le temps requis pour les effectuer. Ces données recueillies permettent de combler les informations requises pour une meilleure planification des effectifs IPO au Québec et d'offrir une méthode d'aide à la décision pour la répartition des effectifs. Cette étude a permis d'identifier 29 interventions jugées prioritaires par les IPO pour prendre soin des patients atteints de cancer ainsi que le temps nécessaire pour les effectuer selon le jugement professionnel des IPO. Ces résultats appellent au moins deux enjeux fondamentaux dans le contexte d'une planification des effectifs pour ce groupe professionnel. Le premier enjeu est le fait que le temps requis, selon les IPO, pour intervenir auprès de ces patients fait en sorte que la demande en soins infirmiers dépasse largement l'offre de service actuelle basée sur le nombre de nouveaux cas par année au Québec. Par conséquent, certains patients n'auront pas accès à ce service, car l'offre est plus limitée que la demande. Un deuxième enjeu est en lien avec l'efficacité du rôle tel que perçu par les IPO.

Le premier enjeu, le fait que le nombre actuel d'IPO ne permet pas de répondre aux objectifs fixés, soit que $70 \%$ des patients atteints d'un cancer aient accès à une IPO, appelle à mettre en place certaines stratégies. Les options sont, cependant, assez limitées. L'une de ces options est d'augmenter l'offre de service en recrutant plus d'infirmières pivots. Cette option a été mise en place, mais le nombre d'embauches ne correspondra jamais aux besoins identifiés dans cette étude. Une autre option est d'augmenter l'offre de service en augmentant la productivité (Birch et al., 2007). Le temps consacré aux tâches connexes inhérentes à l'organisation du travail (tâches non cliniques) est comparable à celui déjà rapporté dans la littérature (Hendrich, Chow, Skierczynski et Lu, 2008). Dans ce contexte, la capacité d'augmenter l'offre de service par une augmentation de la productivité est possible, mais offrira des gains limités.

Une des stratégies les plus porteuses pour faire face à ce défi se situe probablement au niveau de l'identification des patients pour lesquels les interventions de l'IPO sont le plus bénéfiques étant donné son rôle et sa contribution particulière. L'identification de ces patients peut être faite en ciblant les clientèles les plus à risque et par une meilleure connaissance de l'impact des interventions des IPO.

Certaines échelles pour identifier les clientèles à risque existent dans d'autres domaines, notamment en gériatrie (Hoogerduijn et al., 2007; Bissett, Cusick et Lannin., 2013). Ce besoin de bien identifier les clientèles à risque constitue d'ailleurs l'une des premières recommandations issues d'une récente revue systématique sur le rôle de coordonnateur de soins (Conway, O’Donnell et Yates, 2017). Plus spécifiquement, les auteurs recommandent l'adoption d'un système qui facilite l'identification des clientèles à risque au moyen de divers facteurs (personnes âgées, comorbidités, littératie, etc.).
Dans le but d'identifier ces critères, un exercice de consensus professionnel avec la participation de 70 infirmières pivots a été mené lors de la conférence annuelle de l'Association québécoise des infirmières en oncologie (AQIO) en 2013. À la question sur les critères à considérer pour déterminer quels sont les patients/familles qui ont besoin d'un suivi de l'IPO, les résultats ont dégagé les thèmes suivants : stade de la maladie, comorbidité, situation psychosociale et traitements multiples. Les critères retenus pour terminer le suivi présupposent un suivi à long terme (décès, transfert dans un autre centre ou aux soins palliatifs) qui est inhérent à la conception actuelle du rôle d'IPO.

Les informations actuelles sur les facteurs prédictifs ou sur les outils de dépistage (repérage) qui identifient les besoins prioritaires d'une personne atteinte de cancer sont majoritairement liées à trois thèmes, soit la détresse émotionnelle, les symptômes associés au cancer et ses traitements, et le statut fonctionnel (Abernathy et al., 2010; Breen et al., 2012; Carlson, 2012). Ceux-ci sont très similaires à ceux identifiés par les IPO lors de l'atelier.

Dans le plan d'action de la Direction générale de cancérologie 2016-2017 du MSSS, sous le point « optimiser l'accès des personnes atteintes de cancer à l'IPO », le développement de trois outils cliniques est prévu, soit la demande de consultation, la grille de priorisation des demandes et le guichet d'accès avec une équipe de triage. Les travaux cités plus haut ont contribué à l'élaboration de ces outils. L'efficacité de ces outils à identifier les clientèles prioritaires devra être évaluée lors de leur déploiement.

Cette meilleure priorisation et, en parallèle, la meilleure connaissance des bénéfices associés à ce rôle pour les patients constituent probablement les deux axes de recherche à poursuivre afin de maximiser ce rôle au cours des prochaines années. Dans une étude (Paskett, 2011), l'efficacité de la navigation semble plus importante lors de la période de dépistage du cancer que lors du diagnostic et du suivi des traitements; ou encore elle est reconnue plus efficace pour l'adoption de comportements de santé notamment en phase de dépistage et de suivi des traitements (Ali-Faisal, Colella, Tracey et al., 2016). Une autre étude (Bellomo, 2014) rapportait que les patients ayant un suivi d'une IPO (« Nurse Navigator ») rapportaient une meilleure continuité des soins et satisfaction.

Il s'avère que le modèle québécois de déploiement des IPO a mis surtout l'emphase sur le suivi longitudinal dans le cadre de l'annonce du diagnostic et des traitements tel que démontré par le temps consacré pour le suivi durant les traitements de chimiothérapie. Les recherches doivent se poursuivre afin de mieux documenter quelles interventions sont les plus pertinentes dans un modèle de navigation dans le suivi des traitements.

Si la nécessité de mieux documenter l'impact de la navigation en cours de traitement sur la santé des patients est toujours présente, ce modèle semble cependant avoir un impact sur l'expérience de soins vécue par les patients. Il ressort de la dernière enquête québécoise sur l'expérience des patients (Dubé-Linteau, 2014) que les patients ayant accès à une infirmière pivot sont plus susceptibles d'évaluer comme excellente 
ou très bonne la qualité des soins et services reçus dans leur ensemble. Qui plus est, cette différence positive est présente pour l'ensemble des six dimensions de la qualité étudiées, 1) soit l'accessibilité; 2) la coordination et la continuité; 3) le soutien émotionnel; 4) l'information, la communication et l'éducation; 5) le confort physique; et 6) le respect des préférences du patient. Attieh et Loiselle (2018) obtiennent des résultats très similaires dans leur étude récente qui compare le suivi des patients avec et sans un suivi par une IPO. Les résultats démontrent encore une fois une différence significative tant au niveau de leurs soins que de leur satisfaction.

\section{Limites}

Quelle que soit l'approche choisie, il demeure toujours difficile d'estimer de façon précise le temps nécessaire pour prodiguer des soins infirmiers de qualité. À titre d'exemple, les facteurs organisationnels de chacun des milieux n'ont pas été considérés. Une infirmière qui utilise un dossier-patient informatisé influencera le temps requis pour la documentation. Ces facteurs peuvent peut-être expliquer en partie pourquoi le temps requis pour accomplir certaines interventions infirmières n'a pas atteint le niveau de consensus choisi dans le

\section{RÉFÉRENCES}

Abernathy, A.P et al. (2010). Utility and use of palliative care screening tools in routine oncology practice. Cancer Journal, 16(5), 444-460.

Ali-Faisal, S.F., Colella, T.J.F., Medina-Jaudes, N., Benz, L. (2016) Do patient navigators improve cancer care utilization behaviors? A meta-analysis. Journal of Oncology and Survivorship, 7(9).

Ali-Faisal, S.F., Colella, T.J.F., Medina-Jaudes, N., Scott, L.B. (2017). The effectiveness of patient navigation to improve healthcare utilization outcomes: A meta-analysis of randomized controlled trials. Patient Education and Counseling, 100(3), 436-448.

Attieh, S., Loiselle, C., Thomas, D., Ehrler, A., Allard, M., Tardif, L., \& Rouseau, C. (2018). The pivot nurse navigator role is linked to significantly higher quality of the patient reported cancer experience. In Preparation.

Bellomo, C. (2014). The effect of navigation intervention on continuity of care and patient satisfaction of patient with cancer. Journal of Oncology Navigation \& Survivorship, 5(6).

Birch, S., Kephart, G., Tomblin-Murphy, G., O'Brien-Pallas, L., Alder, R., \& MacKenzie, A. (2007). Human resources planning and the production of health: A needs-based analytical framework. Canadian Public Policy, 33, S1-S16.

Biron, A.D., \& Saucier, A. (2012). Infirmière pivot en oncologie : Estimation des besoins en effectifs selon la cible ministérielle du Québec. Direction québécoise de cancérologie, Montréal.

Bissett, M., Cusick, A., \& Lannin, N.A. (2013). Functional assessments utilised in emergency departments: a systematic review. Age and Ageing, 42(2), 163-172.

Boulkedid, R., Abdoul, H., Loustau, M., Sibony, O., \& Alberti, C. (2011). Using and reporting the Delphi method for selecting healthcare quality indicators: A systematic review. PloS One, 6(6), e20476.

Breen, S., Ristevski, E., \& Regan, M. (2012). Enabling supportive care screening and evidence-based referrals for patients with cancer: Patient acceptability and clinician implementation of the Supportive Care Resource Kit (SCRK). Australian Journal of Cancer Nursing, 13(1), 20. cadre de cette étude. Malgré ces limites, l'approche utilisée, soit par le consensus professionnel, représentait la meilleure option pour estimer les besoins en effectifs IPO considérant l'absence de données probantes sur la relation entre le nombre d'IPO déployées et les résultats sur la santé de la population desservie.

\section{CONCLUSION}

Toutes les étapes franchies en utilisant la technique du groupe nominal et la méthode Delphi ont permis détablir un consensus professionnel avec un groupe d'experts et de valider ce consensus avec un échantillon plus large pour ainsi générer des données quant au temps requis par une IPO pour exécuter les 29 interventions jugées nécessaires auprès d'un patient type qui traverse différentes phases de la maladie et différentes phases de la trajectoire de soins. Ces résultats viennent soutenir la prise de décision dans la planification des effectifs au niveau local, régional et provincial, et ce, en assurant une participation active des professionnels dans les décisions qui les touchent directement dans leurs efforts d'offrir des services de qualité à la population.

Canadian Cancer Society's Advisory Committee on Cancer Statistics (2014). Canadian Cancer Statistics 2014. Toronto, ON: Canadian Cancer Society.

Carlson, L.E., Waller, A., \& Mitchell, A.J. (2012). Screening for distress and unmet needs in patients with cancer: Review and recommendations. Journal of Clinical Oncology, 30(11), 1160-1177.

Comité consultatif des infirmières en oncologie (2008). Rôle de l'infirmière pivot en oncologie. Québec : Direction de la lutte contre le cancer [DLCC], MSSS.

Comité de l'évolution de la pratique infirmière en oncologie (CEPIO) (2005). Pour optimiser la contribution des infirmières à la lutte contre le cancer. Québec : Programme québécois de lutte contre le cancer, Ministère de la santé et des services sociaux (MSSS).

Conway, A., O’Donnel, C., Yates, P. (in press). The effectiveness of the nurse care coordinator role on patient-reported and health service outcomes : A systematic review. Evaluation Q 2 the Health Professions.

Direction de la lutte contre le cancer (2011). Rapport d'activités 2010 2011. Québec : Direction des communications du ministère de la Santé et des Services sociaux du Québec.

Direction de la lutte contre le cancer (2007). Rapport d'activités 20062007. Québec : Direction des communications du ministère de la Santé et des Services sociaux du Québec.

Dubé-Linteau, A. (2014). Enquête québécoise sur la qualité des services de lutte contre le cancer 2013 : L'infirmière pivot en oncologie et l'expérience patient. Québec : Institut de la statistique du Québec.

Evans, C., Rogers, S., \& McGraw, C. (2004). Using consensus methods to establish multidisciplinary perspectives on research priorities for primary care. Primary Health Care Research and Development, 5.

Jones, J., \& Hunter, D. (1995). Qualitative Research: Consensus Methods for Medical and Health Services Research. BMJ, 311(5 August).

Kohli, E., Ptak, J., Smith, R., Taylor, E., Talbot, E.A., \& Kirland, K.B. (2009). Variability in the Hawthorne Effect with regard to hand hygiene performance in high and low performing inpatient care 
units. Infection Control and Hospital Epidemiology, 30(3), 222-225. doi:10.1086/595692

Fillion, L., Aubin, M., de Serres, M., Robitaille, D., Veillette, A.M., \& Rainville, F. (2010). The process of integrating oncology nurse navigators into joint hospital-community local teams. Canadian Oncology Nursing Journal, 20(1), 30-35.

Fillion, L., de Serres, M., Cook, S., Goupil, R.L., Bairati, I., \& Doll, R. (2009). Professional patient navigation in head and neck cancer. Semin Oncol Nurs, 25(3), 212-221.

Harvey N., \& Holmes, C.A. (2012). Nominal group technique: An effective method for obtaining group consensus. International Journal of Nursing Practice, 18(2), 188-194.

Hasson, F., \& Keeney, S. (2011). Enhancing rigour in the Delphi technique research. Technological Forecasting and Social Change, 78(9), 1695-1704.

Hendrich, A., Chow, M.P., Skierczynski, B.A., \& Lu, Z. (2008). A 36-hospital time and motion study: how do medical-surgical nurses spend their time? The Permanente Journal, 12(3), 25.
Hoogerduijn, J.G., Schuurmans, M.J., Duijnstee, M.S. De Rooij, S.E., Grypdonck, M.F. (2007). A systematic review of predictors and screening instruments to identify older hospitalized patients at risk for functional decline. Journal of Clinical Nursing, 16(1), 46-57.

Paskett, E.D., Harrop, J., \& Wells, K.J. (2011). Patient navigation: An update on the state of the science. CA: A Cancer Journal for Clinicians, 61(4), 237-249.

Plante, A., \& Joannette, S. (2009). Monteregie Comprehensive Cancer Care Centre: Integrating nurse navigators in Monteregie's oncology teams: Oone aspect of implementing the Cancer Control Program-Part 1]. Can Oncol Nurs J, 19(1), 13-18.

Presser, S., Couper, M.P., Lessler, J.T., Martin, E., Martin, J., Rothgeb, J.M., \& Singer, E. (2004). Methods for testing and evaluating survey questions. Public Opinion Quarterly, 68(1), 109-130.

Spetz, J., Donaldson, N., Aydin, C., \& Brown, D.S. (2008). How many nurses per patient? Measurements of nurse staffing in health services research. Health Services Research, 43(5p1), 1674-1692. 